\title{
Políticas públicas dirigidas a personas consumidoras de drogas: Estrategias para la desestigmatización y la promoción de los derechos humanos
}

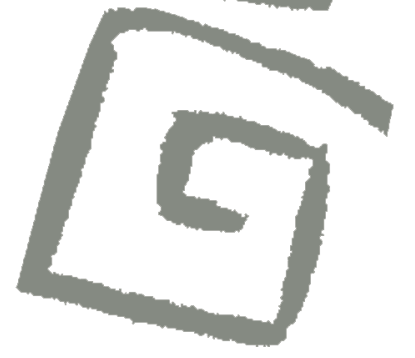

\author{
Public policies for people who use drugs: Strategies for \\ the elimination of stigma and the promotion of human \\ rights
}

Antoniu Llort Suárez , Rafael Clua-García²

${ }^{1}$ Doctor en Antropología Social y Cultural. Investigador, Medical Anthropology Research Center, Universitat Rovira i Virgili, Tarragona, España. $\triangle$ (iD)

${ }^{2}$ Autor de correspondencia. Doctor en Antropología Social y Cultural. Profesor asociado, Facultad de Ciencias de la Salud de Manresa, Universitat de Vic - Universitat Central de Catalunya, Manresa, España. $\triangle$ iD
RESUMEN El objetivo de este artículo es aportar ideas teóricas y prácticas para la mejora de las políticas de drogas actuales, basadas en un modelo biológico de la enfermedad y en la criminalización de las personas que consumen sustancias ilegales. Para ello, en primer lugar, se ofrecen alternativas para superar las bases científicas biologicistas centradas en los postulados neurocientíficos, que apoyan la idea de que en el cerebro se materializa la etiología de la adicción, y apoyar los modelos basados en las ciencias sociales, en los que el contexto y el aprendizaje social juegan un papel relevante para la descripción, el abordaje y la gestión de los diferentes usos de sustancias psicoactivas. En segundo término, se ofrecen modelos y propuestas epistemológicas, desde una perspectiva práctica, para sostener o implementar políticas y programas acorde con un abordaje más sostenible, basado en la eliminación del estigma y la promoción de la participación política de personas consumidoras de sustancias ilegales, es decir, unas políticas sobre drogas basadas en derechos humanos.

PALABRAS ClaVeS Políticas Públicas de Salud; Reducción del Daño; Estigma Social; Derechos Humanos; Antropología Médica.

ABSTRACT This paper aims to provide theoretically informed practical proposals for the improvement of current drug policies, which are based on a biological model of disease and the criminalization of people who use drugs. First, we present alternatives to a biologically-oriented scientific conception centered around neuroscientific postulates, which support the idea that the etiology of addiction materializes in the brain, in favor of models based on the social sciences where context plays a relevant role in the description and management approaches regarding different uses of psychoactive substances. Second, epistemological models and proposals are offered from a practical perspective to sustain or implement policies and programs in accordance with a more sustainable approach based on the elimination of stigma and the promotion of political participation of people who use drugs. In short, drug policies based on human rights.

KEY WORDS Public Health Policy; Harm Reduction; Social Stigma; Human Rights; Medical Anthropology. 


\section{INTRODUCCIÓN}

Para entender el estado actual de las políticas de drogas, debemos invocar el movimiento prohibicionista gestado en EEUU a principios del siglo XX que se apoyó en el rechazo a las minorías étnicas, su supuesta criminalidad asociada al consumo de sustancias psicoactivas, y un sustrato religioso protestante radical como explicación del fenómeno. En la actualidad, dichas políticas continúan fundamentándose en bases científicas biologicistas y legales represivas. El enfoque biologicista pretende desahuciar las teorías sociales que explican el consumo de sustancias, centrándose en el análisis del contexto y su interacción con las diferencias individuales y variabilidad de las sustancias. El objetivo es descartar la posibilidad de modelos comprensivos alternativos, que contemplen la posibilidad de una gestión responsable del consumo de sustancias y que rechacen una guerra contra las drogas. La investigación en ciencias sociales ayuda a mostrar-mediante la profundización en diferentes contextos a lo largo de la historia- cómo el diseño de las políticas de drogas y los instrumentos de la política están determinados por los mismos valores políticos, en particular, por la noción específica de ciudadanía y el papel del Estado que existe en cada país. Estos sistemas de gobernanza incluyen: organización de sistemas políticos, las formas en que operan las instituciones, los equilibrios de poder que se estabilizan entre instituciones en un período de tiempo determinado, las tradiciones legales y administrativas específicas, la capacidad de influir en el poder por parte de los movimientos sociales, y la mayor o menor legitimidad de sus acciones ${ }^{(1)}$.

En los últimos años, se ha evidenciado la dudosa efectividad de este enfoque represivo que, lejos de conseguir sus metas, produce daños colaterales aún mayores para la salud pública ${ }^{(2)}$. Algunas de las consecuencias del modelo prohibicionista que goza de un estatus hegemónico son: las desproporcionadas penas de prisión, las muertes en nombre de la lucha contra el narcotráfico, el miedo a las medidas represivas como obstáculo para el acceso a los tratamientos, la exposición a violencia estructural, la discriminación y la negación del derecho a la salud, el fortalecimiento de los grupos armados, las estrictas regulaciones para el uso de opiáceos en el ámbito médico o la tortura y abusos sexuales ${ }^{(3)}$.

En el marco de las políticas prohibicionistas, los problemas sociales y de salud entre las personas usuarias de drogas han sido intensificados en sociedades con un estado de bienestar debilitado y modelos políticos neoliberales que han favorecido las escenas abiertas de consumo u opacas en las que se integran poblaciones vulnerables que realizan prácticas ocultas. A estos "entornos de riesgo"(4) acuden personas en situación de exclusión social y pobreza, en las que el consumo de drogas es moldeado por factores sociales, estructurales y ambientales con la función de aliviar el sufrimiento social ${ }^{(5,6,7)}$. En dichos espacios ocultos, como las shooting galleries y crack house en EEUU y Australia ${ }^{(8,9)}$, los fumaderos y "cracolandias" en Latinoamérica ${ }^{(6,10)} \mathrm{O}$ "chutaderos" y pisos de venta y consumo en el sur Europa $^{(7,11)}$, las personas usuarias de drogas se exponen a diversos daños sociales y a la salud y a diferentes tipos de violencia como consecuencia de la marginación y la estigmatización socioespacial marcadas por los imperativos de la norma social.

En los diversos "entornos de riesgo"(4) tienen mayor presencia usuarios que consumen cocaína y heroína, entre otras drogas, por vía parenteral y pulmonar. En estos lugares, caracterizados por la insalubridad, falta de seguridad y las limitaciones materiales del entorno, los usuarios llevan a cabo prácticas de riesgo social y a la salud. En el consumo de las diversas presentaciones de opioides (heroína, fentanilo, etc.) y cocaínas (crack, paco, etc.), mediante diferentes métodos de consumo por vía pulmonar (pipas, latas, etc.) e inyectado (intravenoso, subcutáneo, etc.), las personas consumidoras presentan problemas de salud física, relacionados con la pérdida de peso; bajo cuidado personal; problemas dermatológicos; infecciones de transmisión sanguínea (VIH y hepatitis B y C) por compartir tubos improvisados para fumar o compartir jeringuiIlas y otros utensilios para la preparación de la 
inyección; sobredosis de opioides o reacciones adversas de cocaína; problemas cardiovasculares y pulmonares -más acentuados en el uso por vía pulmonar-; así como cuadros de salud mental, tales como depresión, síntomas psicóticos y pensamiento suicida ${ }^{(5,7,10,11,12,13,14)}$.

Las prácticas de consumo ocultas se producen en los márgenes, en "territorios psicotrópicos"(15), espacios urbanos caracterizados por la pobreza y la segregación, a los que acuden los usuarios de drogas para adquirir y ritualizar el consumo de drogas en condiciones extremas y bajo el control policial, que constatan el fracaso de las políticas de intervención social y de drogas. En dichos entornos, los usuarios de drogas sufren violencia simbólica, cotidiana y estructural ${ }^{(5,16,17,18)}$. La violencia simbólica se caracteriza por la autoculpa y estigma entre los usuarios de drogas, al integrar el sufrimiento y los daños del consumo como un "castigo" justificable. La violencia cotidiana se refiere a la normalización de la violencia y el sufrimiento entre los usuarios y otros agentes implicados, que impulsa relaciones de poder y género, representadas mediante violencia física en la cotidianidad de las escenas de consumo. La violencia estructural se relaciona con las normas sociales que infligen a los usuarios de drogas relacionadas con la criminalización de las drogas, que implica violencia policial y limitaciones en el acceso a servicios sociosanitarios.

Por tantos motivos, y a partir de nuestra experiencia como profesionales en servicios de atención a las drogodependencias y como investigadores de políticas públicas sobre drogas y su impacto en esta población, en este trabajo queremos reflexionar, teóricamente y de forma aplicada, sobre posibles estrategias para combatir el estigma y fomentar el desarrollo de los derechos humanos entre las personas que usan drogas. Con este propósito, en una primera parte ofrecemos alternativas teóricas para superar las bases científicas biologicistas, apoyándonos en modelos basados en las ciencias sociales que abogan por la relevancia del contexto y los aprendizajes sociales en la gestión y abordaje de los diferentes usos de sustancias psicoactivas. En una segunda parte, ofrecemos una selección de experiencias e investigaciones para implementar políticas y programas sostenibles para mitigar la estigmatización y promover la participación de las personas consumidoras en política públicas que incluyan los derechos humanos.

\section{EL CONCEPTO DE ADICCIÓN, COMO ENFERMEDAD DEL CEREBRO: UNA ENTELEQUIA PROHIBICIONISTA}

Tanto la biomedicina (como paradigma científico) como la idea cultural contemporánea, consideran el uso de sustancias psicoactivas ilegales como una enfermedad o un acto desviado. Tanto el modelo de enfermedad cerebral como el de desviación social, son los modelos dominantes ante la representación sociocultural de este fenómeno, así como legitiman la continuidad de unas políticas públicas que perpetúan y permiten la estigmatización de las personas que consumen este tipo de sustancias. El cuestionamiento del concepto de adicción como enfermedad biológica arguye la posibilidad de que este concepto se trate de un "síndrome culturalmente construido", influenciado por las características sociohistóricas de nuestras sociedades ${ }^{(19)}$. Las distintas aproximaciones, definiciones y clasificaciones apropiadas para lo que conocemos como adicción, no han conseguido llegar a un nivel de consenso suficiente. La adicción, como enfermedad, no surgió de la acumulación natural de descubrimientos científicos $^{(20)}$, sino que fue inventada histórica y culturalmente bajo condiciones específicas, promulgada por actores e instituciones particulares, y se reprodujo por medio de ciertas prácticas discursivas. Según Peele ${ }^{(21)}$, la idea de que la adicción es resultado de un mecanismo biológico específico que bloquea el cuerpo en un patrón invariable de comportamiento está siendo ampliamente discutida mediante una gran gama de pruebas. Aun así, las políticas públicas relacionadas con el uso de sustancias psicoactivas -aunque con la premisa de proteger a la población- desde un análisis complejo, pueden ser más dañinas que beneficiosas. 
Desde otro punto de vista Puerta y Pedrero $^{(22)}$ critican que, en la actualidad, los "adictos" sean etiquetados como "enfermos" por consumir drogas, así como que se les asigne un trastorno mental o cerebral bajo el yugo de la "patología dual", impulsado por psiquiatras con fuertes conflictos de intereses con la industria farmacéutica, generando mayor estigmatización con un sinfín de etiquetas diagnósticas asociadas al uso de drogas. Tomando estas reflexiones, consideraremos que la investigación científica debería focalizar, en mayor medida, sus esfuerzos en la promoción de la autonomía personal de los afectados, la observación de sus estrategias de autorregulación y de reducción de daños y riesgos, y en su relación con el contexto social y no tanto en la búsqueda del origen y el tratamiento biológico del problema.

Actualmente, la idea de que la adicción es una enfermedad cerebral trasciende la psiquiatría y la psicología clásicas y se basa principalmente en los postulados de las neurociencias humanas $y$, especialmente, en la técnica de la neuroimagen. Como principio básico, las neurociencias proponen que todas las manifestaciones de la cultura pueden ser reducidas y explicadas como actividad del cerebro o actividades neurobiológicas ${ }^{(23)}$. Aun así, este campo no está en condiciones de precisar el significado de esos resultados ni de correlacionar la causalidad con la función de las activaciones cerebrales detectadas, ya que la complejidad de la realidad humana trasciende en demasía una prueba clínica, que sí puede ser considerada un interesante nuevo campo de investigación, pero no una prueba concluyente.

\section{Pluralidad frente a singularismo biomédico: distintas formas de entender el concepto de salud y enfermedad}

Las diferentes formas de atención a la enfermedad, que son muchas y muy variadas (pluralismo médico), utilizan diversas técnicas de diagnóstico, detección y tratamiento que, a su vez, están relacionadas con distintas características religiosas, étnicas, económico-políticas, técnicas y científicas, configurando así diferentes formas de atención que, a menudo, no se manifiestan aisladamente, sino que se solapan unas con otras. La biomedicina y otras medicinas profesionales se caracterizan por considerar subalternas la mayoría de estas formas diferenciales, sobre todo y especialmente las actividades denominadas como autoatención, configurando una visión antagónica y excluyente entre estas formas de atención ${ }^{(24)}$. Se desprende de aquí que el consumo de toda sustancia -en cualesquiera de sus múltiples facetas - fuera de la legalidad o del control médico farmacéutico se considerará un uso desviado, patológico o, como veremos a continuación, un saber o práctica a modificar.

Consideramos autoatención, cuidados legos o health lay care a una dimensión estructural que se da en todas las sociedades, también conocida como folk medicine, y que responde a cómo la población o la sociedad entiende, incorpora el concepto de salud y cómo debe usar y qué tipo de respuesta puede o debe obtener. Un buen ejemplo sobre la teorización de un proceso de autorregulación mediante los rituales sociales de consumo de sustancias ilegales es que están orientados al control y a la regulación de la experiencia del consumo de drogas y que ese control se produce de las siguientes maneras: 1) maximizando el efecto deseado de la droga; 2) controlando los niveles de consumo de las drogas; 3) equilibrando los efectos negativos y positivos de las drogas consumidas; 4) previniendo problemas secundarios ${ }^{(25)}$.

La riqueza de los distintos sistemas de salud, entendidos en sentido amplio, y sus distintas concepciones por parte de la población, serán tenidas en cuenta y elaboradas desde la antropología médica por la teoría de los modelos explicativos. Esta subdisciplina de la antropología se adaptará para poder analizar el discurso y las significaciones sobre la salud, la enfermedad, la atención y la prevención en relación con distintos itinerarios asistenciales $\mathrm{y}$, en mayor medida, de itinerarios de consumo. Al mismo tiempo, estos itinerarios, discursos y significados, serán 
analizados consecuentemente como productos históricos y sociopolíticos y en relación con la experiencia vivida a través del cuerpo, como un dispositivo utilizado para la producción de elementos de creación de identidad social y como vehículo para adaptarse a distintas situaciones vitales.

Los sistemas de atención a la salud articulan la enfermedad como un idioma cultural, relacionando creencias sobre las causas de la enfermedad, la experiencia del síntoma, patrones específicos sobre el comportamiento de las enfermedades, decisiones en torno a tratamientos alternativos, prácticas terapéuticas, evaluaciones sobre los resultados terapéuticos, estableciendo relaciones sistemáticas entre estos comportamientos ${ }^{(26)}$. En este sentido, los conceptos de disease e illness que Fabrega ${ }^{(27)}$ presentó como objetos o entidades observables y estáticas conceptualmente serán resignificados no como entidades derivadas de la observación empírica, sino como diferentes maneras de explicar la enfermedad, diferentes construcciones sociales de la realidad. De ahí surge la idea de los explanatory models (modelos explicativos) o formas discursivas para explicar un mismo fenómeno. En cada sector o área social del sistema de atención a la salud, los diferentes modelos explicativos pueden emanar de médicos, pacientes o la familia para explicar cada episodio particular de enfermedad o fenómenos relacionados con la salud. Los modelos explicativos contienen argumentos y descripciones de cualquiera de los siguientes temas, o de todos ellos: etiología de la enfermedad, inicio de los síntomas, patología física, curso de la enfermedad y tratamiento. También están relacionados con distintos sistemas de conocimiento y valores específicos centrados en los diferentes valores o actores del sistema, convirtiéndose así en productos históricos y sociopolíticos. De esta manera, podemos reconocer la paradoja que plantea la influencia de la cultura en la enfermedad como experiencia psicosocial, bajo la influencia de las normas culturales que gobiernan la percepción, valoración y expresión de los síntomas, y determinan las particulares características del rol de la enfermedad que forman parte de los mismos sistemas de salud ${ }^{(26)}$.
Si desde un plano hipotético-teórico trabajamos desde la perspectiva del consumo de sustancias como una enfermedad cerebral y social, podemos considerar que las representaciones de estas se construyen mediante las creencias y expectativas de los pacientes sobre una enfermedad o enfermedad somática (síntoma). Las representaciones de enfermedades son fundamentales para las diferentes teorías de la autorregulación ${ }^{(28,29)}$. La teoría de la autorregulación postula que la enfermedad y las representaciones que determinan la valoración de esta establecen que los comportamientos relacionados con los procesos de salud pueden desencadenarse como resultado de procesos cognitivos y emocionales ${ }^{(30)}$.

Por otra parte, la generalización y traducción de problemas sociales en categorías médicas y psiquiátricas legitiman los modelos verticales de salud pública que, lejos de basarse en el conocimiento y las potencialidades locales para la gestión, priorizan la comunicación vertical, unilineal de carácter monológico. Este modelo se caracteriza por lanzar iniciativas informativas básicas en educación para la salud y prevención (modelo muy utilizado en el ámbito de las drogas a nivel mundial) tomando como modelo "el prototipo de un sujeto racional en la toma de decisiones, limitan el conocimiento de la realidad local y, en consecuencia, el abastecimiento de una relación dialógica"(31).

\section{ENTORNOS PARA COMBATIR LA CRIMINALIZACIÓN Y LA VIOLENCIA: DISTINTAS RESPUESTAS A UN MISMO FENÓMENO}

En los primeros años, la respuesta a la alarma social generada por el fenómeno del consumo problemático de sustancias psicoactivas se articuló en el marco de los llamados programas libres de drogas. En paralelo, se generó un incipiente cuestionamiento de estos programas: se propusieron aproximaciones terapéuticas que no priorizaban la abstinencia y sí proponían un acercamiento a la persona, a sus relaciones y a su entorno 
desde una posición de mayor respeto hacia los individuos y una comprensión de su realidad.

La voluntad de fondo consistía en minimizar daños a partir de un rol más activo y participativo de los destinatarios de los servicios de atención ${ }^{(32,33)}$. Se propone la combinación de un marco epistemológico ecosistémico con el pragmatismo de la actuación de la teoría de reducción de daños y riesgos ${ }^{(33,34)}$. Es por ello, que lo más adecuado, a la hora de dar respuesta a las demandas asistenciales, es concebirlas en un complejo mapa de puntos de contacto e itinerarios múltiples. Estos permiten diseñar recorridos únicos para cada persona, y atienden a sus necesidades a partir de la definición de unos objetivos, en función de los aspectos predominantes de la demanda del sujeto y de sus posibilidades de obtener respuestas deseadas. Para acercarse o tender a esta forma de abordar las relaciones basadas en derechos fundamentales, es importante que el sistema asistencial acepte que las personas consumidoras tienen la última palabra en el planteamiento de cambio. Para ello podemos intervenir desde diferentes niveles. No obstante, para lograr coherencia y articulación, es fundamental la redacción, en coparticipación, de planes de acción de índole comunitaria sobre drogas.

La estigmatización y criminalización hacia las personas usuarias de drogas conlleva a perseverar en la apuesta de las estrategias de reducción de daños para continuar defendiendo los derechos humanos y el acceso a la atención social y a la salud de esta población ${ }^{(7,17)}$. Incluso, si se produjera una marcha atrás en las políticas prohibicionistas y se llevaran a cabo acciones para la despenalización y regulación del uso de drogas, sería necesario la promoción de intervenciones específicas para mitigar las desigualdades sociales y estructurales entre los usuarios de drogas ${ }^{(17)}$. En el marco actual, han surgido diversos espacios y organizaciones con el objetivo de combatir las consecuencias negativas del prohibicionismo y las fuerzas sociales y estructurales que vulneran los derechos y generan violencia entre los usuarios de drogas inmersos en "entornos de riesgo"(4). Entre estos espacios, destacamos las asociaciones de usuarios de drogas y los centros sociosanitarios que integran programas de reducción de daños.

Estos espacios han tenido impacto para mejorar la calidad de vida y fomentar el compromiso en la lucha de intereses entre las personas consumidoras de sustancias psicoactivas, a promover la normalización del uso de drogas con el fin de consolidar procesos de desestigmatización, y a asesorar y gestionar intervenciones, estableciendo un diálogo con profesionales y técnicos para emprender acciones más adaptadas a las necesidades de las personas consumidoras de drogas ${ }^{(35,36)}$. De este modo, los consumidores participan como expertos y como agentes válidos para promover el acercamiento de otros usuarios a servicios sociosanitarios, acompañar en los procesos de salud-enfermedadatención y a movilizar en la comunidad acciones educativas, culturales y preventivas para una gestión normalizada del fenómeno del uso de drogas ${ }^{(37)}$. En este sentido, las fuerzas colaborativas entre personas destinatarias de servicios y profesionales han promovido la creación de servicios de reducción de daños autogestionados o con gestión mixta, con impacto para empoderar a las personas usuarias y crear espacios para combatir las consecuencias sociales y negativas para la salud, generadas en escenas abiertas de consumo.

Siguiendo a Van Dam ${ }^{(38)}$-quien realizó una gran síntesis de la proliferación de estos movimientos organizados en Europa y en los cuales participó activamente-, en 1977, en Holanda, se crearon los primeros grupos de activistas de personas que consumían heroína -también Ilamados grupos de interés- como la Rotterdam Junkie Union (RJU), compuesta por usuarios activos y liderada por Nico Adriaans, y la Medical-social service for heroin users (MDHG), formada por usuarios, familiares, trabajadores sociales y personas que sentían interés. Estos no estaban de acuerdo con las respuestas políticas y sociales que se estaban dando con respecto al consumo de heroína. Este fenómeno se estaba convirtiendo en un problema social grave, sobre todo por la propagación de enfermedades entre personas que se inyectaban drogas. 
En 1980 ya existían quince asociaciones relacionadas con la defensa de los consumidores de drogas en los Países Bajos y, posteriormente, grupos de consumidores empezaron a organizarse por toda Europa hasta la creación en 2005 de la International Network of People who Use Drugs (INPUD), organización que centraliza a nivel internacional la lucha por los derechos de las personas que consumen drogas ilegales.

Actualmente, entre las experiencias lideradas por activistas consumidores cabe resaltar las acciones realizadas por Vancouver Area Network of Drug Users (VANDU), una organización autogestionada por personas que consumen drogas en el Downtown Eastside de la ciudad de Vancouver (Canadá) desde 1998. VANDU cuenta con cerca de 1.000 integrantes y desde su creación han emprendido acciones para dar cobertura al consumo por vía parenteral y pulmonar y de advocacy para impulsar y extender programas de reducción de daños en las políticas locales. Entre sus iniciativas se han realizado intervenciones de proximidad (outreach) para contactar con usuarios en vía pública; la instalación de una sala de consumo supervisado no autorizada para usuarios con problemas para autoinyectarse, gestionada por pares entrenados para asistir el consumo de forma higiénica y segura; y la acomodación de un pequeño espacio para el consumo de crack en sus instalaciones, como alternativa para escapar de la violencia callejera y superar las barreras de acceso a las salas de consumo higiénico oficiales en las que solo es posible el consumo inyectado y la ley no permite la asistencia de la inyección, entre otras acciones ${ }^{(18,39)}$. Cabe también destacar, especialmente, los enfoques feministas acaecidos recientemente, que permiten romper con la lógica más allá del prohibicionismo, un buen ejemplo de ello es el proyecto Metzineres exclusivo para mujeres y personas de género disidente, en múltiples situaciones de vulnerabilidad, nacido en 2017 en Barcelona, formado por un equipo transdisciplinar, que incluye a las personas que consumen sustancias sin diferenciaciones, ofreciendo actividades terapéuticas, culturales y de ocio y que impulsa acciones para una reforma de las políticas de drogas desde una perspectiva feminista no heteronormativa ${ }^{(40)}$. La International Network of People who Use Drugs ya incluía esta aproximación no patriarcal, creándose redes paralelas como la International Network of Women Who use Drugs (INWUD). También surgen experiencias europeas de mujeres y personas de géneros disidentes como estrategia que, por primera vez, permite debilitar el sistema prohibicionista, pero aún más el sistema patriarcal y heteronormativo que los sustenta, véase también el caso de la Red de Mujeres Antiprohibicionistas (REMA) o Mujeres cannábicas en España.

En el plano de la atención sociosanitaria se han desarrollado programas de reducción de daños y la promoción de entornos más seguros $^{(17)}$. A finales de la década de 1980, desde Europa y EEUU se han impulsado diversos programas con impacto para reducir los daños sociales y a la salud entre los usuarios de drogas en situación vulnerable ${ }^{(33,41,42,43,44)}$. Entre los programas e intervenciones más extendidas y evaluadas positivamente a nivel global, se encuentran los programas de intercambio de jeringuillas, que tienen el objetivo de distribuir material para el consumo higiénico y seguro de drogas por vía parenteral y pulmonar; los programas de sustitución con opioides, que consiste en la dispensación controlada de opioides (metadona, buprenorfina, morfina, etc.) con el objetivo de regular el consumo de este tipo de sustancias y reducir el riesgo de transmisión de enfermedades infectocontagiosas (VIH y hepatitis B y C) y las sobredosis, mejorar la calidad de vida y disminuir las actividades delictivas asociadas; las salas de consumo higiénico, que son espacios para el consumo de drogas bajo supervisión de profesionales entrenados para la asistencia de sobredosis u otras complicaciones y la atención médica y de servicios sociales; así como espacios de refugio (drop-in), que funcionan como entornos para el reposo, la alimentación y la higiene personal, entre otras actividades de atención sociosanitaria y lúdica para usuarios en situación vulnerable 
y sin hogar. En diversas ciudades europeas y estadounidenses, cada vez son más los centros sociosanitarios que integran programas de reducción de daños y tratamientos para una atención holística de los usuarios de $\operatorname{drogas}^{(11,17,45)}$.

En diversos estudios etnográficos y cualitativos han explorado el impacto de los servicios de reducción de daños para reducir la vulnerabilidad, los déficits sociosanitarios y la violencia entre los usuarios de drogas. En trabajos realizados en centros de reducción de daños que disponen de salas de consumo higiénico, se concluye que los usuarios integran mensajes preventivos y reciben atención sanitaria para reducir las infecciones de transmisión sanguínea y las sobredosis, así como para reducir prácticas de riesgo y desechar material de consumo en vía pública y otros entornos de riesgo ${ }^{(11,45,46)}$. En un estudio realizado en una salas de consumo higiénico exclusiva para mujeres en la ciudad de Vancouver, las participantes respondían positivamente a la seguridad e higiene provista en estas instalaciones ${ }^{(47)}$. Además, en estos dispositivos, los usuarios sienten que pueden recibir ayuda y ser conectados con otros servicios de atención social y de salud ${ }^{(48,49,50)}$. Los centros de reducción de daños que disponen de zonas de reposo y salas de consumo higiénico son percibidos como entornos seguros para escapar de la violencia y otros riesgos sociales asociados al consumo en vía pública ${ }^{(17)}$. En investigaciones realizadas en salas de consumo higiénico de Vancouver y diversas ciudades europeas, los participantes refieren que acudir a estos espacios es una forma de evitar la violencia cotidiana entre usuarios, el estigma, y la violencia estructural de la intervención policial ${ }^{(11,17,49,51)}$. En estudios realizados con mujeres $^{(16,17,47)}$, se concluye que las salas de consumo higiénico significaron un lugar de refugio y reposo alternativo para mediar las relaciones de poder y abusos de compañeros en la vía pública y fortalecer la agencia para realizar la práctica de consumo.

\section{Accesibilidad y participación de las personas consumidoras de sustancias psicoactivas ilegales en estrategias de reducción de daños}

Pese a que los objetivos de los programas de reducción de daños suelen estar bien definidos, las personas consumidoras de drogas continúan encontrando barreras de acceso a estos dispositivos, relacionadas con fuerzas sociales y estructurales, así como con una falta de servicios para cubrir sus necesidades básicas. En el marco de las políticas prohibicionistas, las barreras de acceso afectan de forma variable según la aplicación de las políticas de reducción de daños en los diversos contextos políticos y geográficos. Dependiendo del grado de tolerancia, la aplicación de la persecución y criminalización permite a los usuarios de drogas un mayor o menor acceso a los servicios sociales y de salud. No obstante, en la mayoría de los contextos geográficos, la principal barrera de acceso se relaciona con el miedo y la intervención de los cuerpos policiales en las proximidades de los servicios de drogas que impiden o dificultan la atención social y a la salud entre los usuarios de drogas ${ }^{(10,11,12,52)}$.

Pero también, los servicios de reducción de daños se han aplicado con el objetivo de reducir la morbimortalidad del uso de drogas, no atendiendo dimensiones sociales, estructurales y ambientales que perseveran la vulnerabilidad entre las personas usuarias de drogas. A menudo, las personas destinatarias señalan no acceder a los servicios sociosanitarios por no tener confianza en los profesionales o por considerar que los servicios que ofrecen no se ajustan a la realidad de las poblaciones específicas, como por ejemplo, los centros específicos no distribuyen material o disponen de espacios para el consumo de crack $^{(10,11,18,53)}$, o en ciudades donde se dispone de centros de reducción de daños con salas de consumo higiénico, estas presentan limitaciones relacionadas con los horarios de apertura, la adhesión a las normas de estos espacios y la difícil interacción con otros usuarios y los profesionales $^{(11,49,54)}$. En el caso de las mujeres 
usuarias de drogas, inmersas en un doble estigma, por romper los roles tradicionales de género y por ser consumidoras de drogas ilegales ${ }^{(16,55)}$, diversos estudios señalan que los servicios de drogas no atienden la especificidad en la discriminación, el estigma y la violencia estructural y de género y que limitaban el acceso a las mujeres ${ }^{(16,47)}$.

Por lo anterior, proponemos acciones para aumentar la adherencia a este tipo de servicios y cubrir necesidades sociales y de salud de las personas consumidoras, y resaltamos la importancia de la participación de personas que consumen sustancias psicoactivas ilegales (de manera problemática o no) en las políticas públicas sobre drogas. Una experiencia muy interesante en este sentido es la propuesta de Coca Regulada Paz Garantizada impulsada desde Colombia(56).

\section{Estrategias de mejora de la accesibilidad, efectividad y cobertura de necesidades básicas}

Para incrementar la accesibilidad y efectividad a los servicios es fundamental eliminar obstáculos situacionales y estructurales para maximizar el impacto de los programas de reducción de daños ${ }^{(4)}$. En este sentido, mientras no se produzcan grandes cambios en las políticas prohibicionistas, es preciso la modificación de leyes locales en contextos geográficos contrarios a las políticas de reducción de daños y a establecer relaciones positivas entre los servicios de reducción de daños, la policía y las personas consumidoras de drogas, que propicien un entorno social para disminuir la criminalización y vulneración de los derechos de los usuarios ${ }^{(4,52)}$. En el medio comunitario son necesarias intervenciones de proximidad (outreach), en las que intervengan profesionales y usuarios expertos (agentes de salud) que conecten a los usuarios con los programas de reducción de daños y servicios en la comunidad.

Para una optimización de los servicios de reducción de daños es necesario que estos inspiren confianza y dispongan servicios que den cobertura a sus necesidades básicas reales. Estudios realizados en centros de reducción de daños con salas de consumo higiénico, señalan que los usuarios aumentaban la adherencia a estos dispositivos en aquellos casos en los que es posible establecer relaciones favorables con los profesionales, comprensivas y no estigmatizantes, y buena interacción con otros usuarios, además de encontrar una gama amplia de cuidados, más allá de la atención esterilizante de la reducción de daños, que incluye estar en un ambiente libre de hostilidad, donde sentirse apoyado y mitigar experiencias negativas relacionadas con los procesos de drogodependencias y la vida en la calle $\mathrm{e}^{(11,48,49,51,57,58)}$. Sin embargo, consideramos que son necesarias transformaciones para poder ofrecer una cobertura esencial que permita mitigar la violencia y la criminalización entre las personas que consumen sustancias ilegales.

En el caso de los consumidores de opioides en situación vulnerable se precisa una mayor regularización y extensión de los programas de prescripción de heroína. En ensayos clínicos con diacetilmorfina inyectable realizados en varias ciudades de Canadá y en Granada (España), los participantes respondían positivamente al tratamiento para mejorar la estabilidad en sus vidas, establecer una discontinuidad con el contexto de las escenas abiertas de consumo, mejorar aspectos sociales como la búsqueda de trabajo y las relaciones familiares, y a mitigar los procesos de exclusión social, así como a mejorar los hábitos alimentarios, la higiene personal y el seguimiento de enfermedades asociadas al consumo ${ }^{(59,60)}$.

En población en situación de calle se propone extender las housing first a las políticas de reducción de daños. Esta práctica se basa en atender a personas sin hogar crónicamente, con enfermedades mentales graves y trastornos por consumo de sustancias. Estudios realizados en EEUU sugieren la necesidad del acceso a este tipo de programas, aplicando criterios inclusivos -sin que necesariamente la abstinencia sea un requisito- $y$ adecuando el tipo de vivienda y los servicios de apoyo necesarios que han evidenciado la recuperación de las condiciones sociosanitarias y la reconexión familiar y cultural de sus beneficiarios $^{(61,62)}$. 
En conclusión, consideramos primordial aplicar los principios básicos de la reducción de daños a la globalidad de las políticas de drogas, y extender estos de forma amplia a otras esferas de la salud y las condiciones sociales de personas que sufren exclusión.

\section{De un modelo represivo a un modelo basado en la agencia de autorregulación}

Con este cometido, es pertinente cambiar los modelos de intervención basados en la abstinencia de drogas hacia modelos que incluyan el "control" o "autogestión positiva" del consumo como posibilidad amparada por ley. Esto implica mitigar del vocabulario profesional concepciones como "adicto", "recaída", "crónico" u otras formas discursivas que estigmatizan a las personas usuarias de drogas ${ }^{(53,63)}$. De hecho, diversos estudios contradicen que el uso de drogas fiscalizadas sea imposible de controlar, siendo más común que poblaciones vulnerables alternen períodos de consumo intenso con consumo de baja frecuencia, incluso mantengan un consumo autorregulado, según las condiciones sociales, estructurales y ambientales ${ }^{(7,11,64)}$. La mayoría de las personas que utilizan drogas fiscalizadas incorporan mecanismos de control con el fin de obtener experiencias positivas del consumo, estableciendo control del uso de la sustancia, con el grupo que se consume y en el ambiente donde este sucede ${ }^{(51,53,63,65)}$.

Por lo tanto, un modelo basado en la autorregulación reconoce las capacidades de los usuarios de drogas, con un impacto positivo para mitigar la estigmatización y promover programas preventivos inclusivos con objetivos más amplios que la abstinencia ${ }^{(53)}$. Por ejemplo, diversos estudios con consumidores de crack en Brasil(13,14,66) señalan la capacidad de los participantes para regular el consumo mediante la combinación de crack con marihuana, con el objetivo de reducir la ansiedad, la excitabilidad y otros efectos psicoestimulantes negativos; disminuir el deseo de consumo de crack y sobrellevar los periodos de abstinencia; así como para mejorar los patrones de higiene, descanso y alimentación y mejorar la calidad de vida. Otros métodos que señalan los consumidores de crack son los de buscar actividades recreativas, ocupacionales o físicas para eludir la ansiedad de consumo y mantener períodos de control o abstinencia $^{(13)}$.

Por último, es necesario señalar que la mayoría de los postulados e intervenciones están basadas en estudios realizados en ambientes clínicos, corriendo el riesgo de realizar generalizaciones centradas únicamente en personas usuarias con consumo intensivo o problemático. En detrimento, los estudios cualitativos, realizados en ambientes naturales, y desde la perspectiva de los usuarios suelen tener poco impacto en la formulación de políticas de drogas ${ }^{(53,67,68)}$. Generalmente, los investigadores de las ciencias cuantitativas subestiman que los estudios que incluyen la perspectiva de las personas consumidoras de sustancias psicoactivas ilegales sean objetivables, replicables y generalizables, pese a seguir procedimientos rigurosos para el estudio del uso de drogas ${ }^{(10,67,69)}$. Sin embargo, la ciencia de carácter cualitativa, proporciona una mayor y más compleja comprensión de las dimensiones micro y macro del uso de drogas, de los significados y representaciones entre los usuarios de drogas y sobre las características del consumo en entornos vulnerables donde sufren daños sociales y a la salud y diferentes tipos de violencia(69,70,71), que pueden ser más útiles para introducir problemas de salud y variables en estudios epidemiológicos ${ }^{(67,69,70)}$, motivo por el que subrayamos la importancia de acudir a las propuestas de este tipo de estudios para una mayor adaptabilidad y credibilidad en las intervenciones específicas.

\section{CONCLUSIONES}

Esperamos haber podido cuestionar la idea de que el consumo de sustancias psicoactivas ilegales pueda ser considerada unilateralmente por los estamentos jurídicos y médicos como una actividad socialmente desviada o como un trastorno o enfermedad mental originado en el cerebro, hecho que 
inevitablemente ha calado en el imaginario colectivo de manera progresiva y muy eficazmente. A pesar de la parcial y no global implantación de las políticas de reducción de daños y riesgos a nivel internacional, las personas que consumen drogas ilegales se mantienen inmersas en procesos de exclusión y represión -explícita o sutilmente- Ilevando a cabo prácticas de consumo ocultas en entornos de riesgo, caracterizados por condiciones sociales, estructurales y ambientales que generan daños sociales y a la salud (estigma, marginación y diferentes tipos de violencia) entre los colectivos más vulnerables o frente a consumos normalizados. Incluso, ante la poco considerada amenaza de la biomedicalización de las propuestas de reducción de daños y riesgos -la cuales progresivamente centran sus esfuerzos en el ámbito estrictamente sanitario- deben ampliar su apuesta influyendo con más fuerza en la esfera política, para hacer frente a la criminalización y promover con más contundencia políticas a favor de los derechos individuales y colectivos con relación al consumo de drogas. Esto permitiría promover legislaciones más mimetizadas con las diferentes realidades sociales y culturales. Para conseguir este objetivo, es indispensable la articulación de los movimientos sociales liderados por representantes de las personas consumidores de sustancias ilegales, familiares y otros afectados, investigadores y profesionales alineados interdisciplinariamente, para presionar a diferentes niveles a las administraciones responsables de sostener -sin autocrítica alguna- las políticas prohibicionistas sobradamente deslegitimadas por sus pobres resultados en relación con sus objetivos: la disminución de la oferta y la demanda.
La eliminación de las barreras de acceso al derecho a la salud, ampliar la cobertura de los servicios de atención, optimizar la participación política -en sentido ampliode las personas consumidores de sustancias psicoactivas ilegales y legales, atender la especificidad y diversidad de cada individuo y de los colectivos consumidores de sustancias y destinatarios de servicios, y la despenalización del consumo mediante la modificación de los convenios internacionales de fiscalización son algunas de las premisas esenciales, aunque quizá no todas, indispensables para la consecución de dichos objetivos. Orientar, en definitiva, una regulación de las sustancias ilegales distinta a la actual. Todas estas premisas deberían tener como punto de partida un conocimiento profundo y complejo de las múltiples realidades y contextos locales o regionales, con sus características culturales propias en colaboración con los avances biomédicos dedicados a la investigación de la salud humana. Solo así, el avance en pequeños contextos y ámbitos (véase el caso de la regulación del cannabis), las pequeñas victorias, frente a un prohibicionismo que se ha erigido globalmente como un monstruo represivo implacable, son la única vía de expandir un movimiento social y político de defensa de las personas consumidores de sustancias ilegales. En definitiva, un abordaje de la cuestión aquí planteada, más sintónica con los derechos humanos en el ámbito de la salud y el derecho a la libre determinación. Al fin y al cabo, el uso de sustancias psicoactivas forma parte de la incuestionable libertad de atender a las vicisitudes de nuestra salud $y$, por ende, la atención a los malestares o imprevistos que las enfermedades biológicas, sociales o culturales nos plantean. 


\section{REFERENCIAS BIBLIOGRÁFICAS}

1. Milhet $\mathrm{M}$, Moloney $\mathrm{M}$, Bergeron $\mathrm{H}$, Hunt G. Introduction: Drug and culture. En: Hunt $G$, Milhet $M$, Bergeron H. (eds.). Drugs and culture: Knowledge, consumption, and policy. Farnham, England: Ashgate; 2011. p. 1-14.

2. Wodak A. Harm Reduction and law: The impact of criminalisation on public health. En: Brigaud T, (dir.). The history and principles of Harm Reduction: Between public health and social change. Paris: Médecins du Monde, Agence Française de Développement; 2013. p. 110-119.

3. McFarland M. Por qué el respeto a los derechos humanos exige reformas a las políticas de drogas: Cómo la criminalización de las drogas destruye vidas, fomenta abusos y subvierte la justicia. En: Informe Mundial 2014 Human Rights Watch. Nueva York: HRW; 2014. p. 42-50.

4. Rhodes T. Risk environments and drug harms: a social science for harm reduction approach. International Journal of Drug Policy. 2009;20(3):193-292.

5. Bourgois $P$, Prince $B$, Moss $A$. The everyday violence of hepatitis $C$ among young women who inject drugs in San Francisco. Human Organization. 2004;63(3):253264.

6. Epele M. Sujetar por la herida: Una etnografía sobre drogas, pobreza y salud. Buenos Aires: Paidós; 2010.

7. Llort A. "Dime dónde consumes y te diré...": Antropología sobre el uso y abuso de cocaína. Perifèria. Revista de Recerca i Formació en Antropologia. 2018;23(1):56-83.

8. Bourgois P. In search of respect: Selling crack in el barrio. 2nd Ed. Cambridge: Cambridge University Press; 2003.

9. Kimber J, Dolan K. Shooting gallery operation in the context of establishing a Medically Supervised Injecting Center: Sydney, Australia. Journal of Urban Health. 2007;84(2):255-266. doi: 10.1007/s11524-006-9145-3.

10. Toledo L, Góngora A, Bastos FIPM. À margem: uso de crack, desvio, criminalizaçãoeexclusão social-uma revisão narrativa. Ciência \& Saúde Coletiva. 2017;22(1):31-42. doi: 10.1590/1413-81232017221.02852016.

11. Clua R. La gestión de placeres y daños: estudio etnográfico sobre el consumo de drogas en vía pública, viviendas y salas de consumo higiénico. Salud Colectiva. 2020;16:e2481. doi: 10.18294/sc.2020.2481.

12. Cortés E, Metaal P. Mercados de cocaína fumable en América Latina y el Caribe: Llamamiento a favor de una repuesta sostenible en materia de políticas. Amsterdam: Transnacional Institute; 2019.

13. Chaves TV, Sanchez ZM, Ribeiro LA, Nappo SA. Fissura por crack: comportamentos e estratégias de controle de usuários e ex-usuários. Revista de Saúde Pública. 2011;45(6):1168-1175. doi: 10.1590/S003489102011005000066.
14. Jorge MSB, Quinderé PHD, Yasui S, Albuquerque RA. Ritual de consumo do crack: aspectos socioantropológicos e repercussões para a saúde dos usuários. Ciência \& Saúde Coletiva. 2013;18(10):2909-2918.

15. Mata S, Fernandes L. Revisitação aos atores e territórios psicotrópicos do Porto: Olhares etnográficos no espaço de 20 anos. Civitas - Revista de Ciências Sociais. 2019;19(1):195-212.

16. Fairbairn N, Small W, Shannon K, Wood E, Kerr T. Seeking refuge from violence in street-based drug scenes: women's experiences in North America's first supervised injection facility. Social Science \& Medicine. 2008;67(5):817823. doi: 10.1016/j.socscimed.2008.05.012.

17. McNeil R, Small W. "Safer environment interventions": a qualitative synthesis of the experiences and perceptions of people who inject drugs. Social Science \& Medicine. 2014;106:151-158. doi: 10.1016/j.socscimed.2014.01.051.

18. McNeil R, Kerr T, Lampkin H, Small W. "We need somewhere to smoke crack": An ethnographic study of an unsanctioned safer smoking room in Vancouver, Canada. International Journal on Drug Policy. 2015;26(7):645652. doi: 10.1016/j.drugpo.2015.01.015.

19. Apud I, Romaní O. La encrucijada de la adicción: Distintos modelos en el estudio de la drogodependencia. Health and Addictions/Salud y Drogas. 2016;16(2):115125.

20. Reinarman C. Addiction as accomplishment: The discursiveconstruction ofdisease. Addiction Research \& Theory. 2005;13:307-320. doi: 10.1080/16066350500077728.

21. Peele S. The Meaning of addiction: Compulsive experience and its interpretation. Lexington: Mass D. C. Heath; 1985.

22. Puerta C, Pedrero E. La falacia de la adicción como enfermedad cerebral. [Internet]. Las Drogas; 2 may 2017 [citado 20 jul 2020]. Disponible en: https:// tinyurl.com/yytoenxt.

23. Vidal F. Todo a «la neuro»: una cocina autodestructiva. Arxiu d'Etnografia de Catalunya. 2019;20:233-251.

24. Menéndez EL. El modelo médico y la salud de los trabajadores. Salud Colectiva. 2005;1(1):9-32. doi: 10.18294/ sc. 2005.1

25. Grund JP, Kaplan CH, DeVries M. Rituales de Regulación: El consumo controlado e incontrolado de las drogas en el marco natural. En: Heather N, Wodak A, Nadelman E, O'Hare P. (eds.) La cultura de drogas en la sociedad del riesgo. Barcelona: Publicaciones Grup Igia; 2004. p. 67-77.

26. Kleinman A. Concepts and a model for comparison of medical systems as cultural systems. Social Science \& Medicine. 1978;12:85-93. doi: 10.1016/01607987(78)90014-5.

27. Fabrega $\mathrm{H}$. The study of disease in relation to culture. Behavioral Science. 1972;17:183-203. doi: 10.1002/ bs.3830170202. 
28. Leventhal $\mathrm{H}$. Findings and theory in the study of fear communications. Advances in Experimental Social Psychology. 1970;5:119-186. doi: 10.1016/ S0065-2601(08)60091-X.

29. Leventhal H, Meyer D, Nerenz DR. The common sense representation of illness danger. En: Rachman S. (ed.) Contributions to medical psychology New York. New York: Pargamon; 1980. p. 17-30.

30. Leventhal H, Diefenbach $M$, Leventhal EA. Illness cognition: using common sense to understand treatment adherence and affect cognition interactions. Cognitive Therapy and Research. 1992;16(2):143-163.

31. Martínez Hernáez Á. Antropología Médica: Teorías sobre la cultura, el poder y la enfermedad. Barcelona: Anthropos; 2008.

32. de Andrés M, Magrí N, Borrás T. Programas de reducción de riesgo. Barcelona: Grup IGIA; 1996.

33. Romaní O. Políticas de drogas: prevención, participación y reducción del daño. Salud Colectiva. 2008;4(3):301-318. doi: 10.18294/sc.2008.347.

34. Borràs T, Sardà A. Cambios sociales, cambios en los tratamientos. Monografia Humanitas. 2005;5:119-137.

35. Llort A, Borràs T. La diferencia que hace la diferencia: La ampliación de la mirada de género e introducción a una teoría del cuerpo, Una estrategia para aprender junt@s. En: Llort A, Borràs T. (coords.) Apuntes para la promoción de intervenciones con pares. Barcelona: Grup Igia; 2013. p. 264-279.

36. Bröring G, Schatz E. (eds.) Empowerment and selforganisations of drug users experiences and lessons learnt. Amsterdam: Foundation Regenboog AMOC Correlation Network; 2008.

37. Llort A. La organización de los usuarios: La reducción de riesgos en el ámbito de la exclusión social. En: Martínez Oró DP, Pallarés J, (eds.). De riesgos y placeres: manual para entender las drogas. Lleida: Milenio; 2013. p. 195-208.

38. Van Dam T. A brief overview about the drug user movement. En: Bröring G, Schatz E, (eds.). Empowerment and self-organisations of drug users experiences and lessons learnt. Amsterdam: Foundation Regenboog AMOC Correlation Network; 2008. p. 57-61.

39. McNeil R, Small W, Lampkin H, Shannon K, Kerr T. "People knew they could come here to get help": an ethnographic study of assisted injection practices at a peer-run 'unsanctioned' supervised drug consumption room in a Canadian setting. AIDS and Behavior. 2014;18(3):473-485. doi: 10.1007/s10461-013-0540-y.

40. Roig A. Metzineres: cobijo y empoderamiento de mujeres que usan drogas sobreviviendo violencias [Internet]. Wola; 15 dic 2019 [citado 24 jul 2020]. Disponible en: https://tinyurl.com/y697sgf7.

41. Rhodes T, Hedrich D. (eds.) Harm reduction: evidence, impacts and challenges. Luxemburgo: European Monitoring Centre for Drugs and Drug Addiction; 2010.
42. Martínez Oró DP. La dimensión sociopolítica de la reducción de daños y de riesgos en el Estado español. Revista Española de Drogodependencias. 2019;44(3):52-66.

43. Fernandes RM, Cary M, Duarte G, Jesus G, Alarcão J, Torre C, Costa S, Costa J, Carneiro A. Effectiveness of needle and syringe Programmes in people who inject drugs - An overview of systematic reviews. BMC Public Health. 2017;17(1):309.

44. Potier C, Laprévote V, Dubois-Arber F, Cottencin O, Rolland B. Supervised injection services: what has been demonstrated?: A systematic literature review. Drug Alcohol Depend. 2014;145:48-68. doi: 10.1016/j.drugalcdep.2014.10.012.

45. Kennedy MC, Karamouzian M, Kerr T. Public health and public order outcomes associated with supervised drug consumption facilities: a systematic review. Current HIV/AIDS Reports. 2017;14(5):161-183. doi: 10.1007/ s11904-017-0363-y.

46. Krüsi A, Small W, Wood E, Kerr T. An integrated supervised injecting program within a care facility for HIV positive individuals: a qualitative evaluation. AIDS Care. 2009;21:638-644. doi: 10.1080/09540120802385645.

47. Boyd J, Lavalley J, Czechaczek S, Mayera S, Kerr T, Maher L, McNeil R. "Bed Bugs and Beyond": An ethnographic analysis of North America's first women-only supervised drug consumption site. International Journal on Drug Policy. 2020;78:102733. doi: 10.1016/j. drugpo.2020.102733.

48. Kerman N, Manoni-Millara S, Cormier L, Cahill T, Sylvestre J. "It's not just injecting drugs": Supervised consumption sites and the social determinants of Health. Drug and Alcohol Dependence. 2020;213:108078. doi: 10.1016/j.drugalcdep.2020.108078.

49. Kappel N, Toth E, Tegner J, Lauridsen S. A qualitative study of how Danish drug consumption rooms influence health and well-being among people who use drugs. Harm Reduction Journal. 2016;13(1):20.

50. Small W, van Borek N, Fairbairn N, Wood E, Kerr $\mathrm{T}$. Access to health and social services for IDU: the impact of a medically supervised injection facility. Drug and Alcohol Review. 2009;28(4):341-346. doi: 10.1111/j.1465-3362.2009.00025.x.

51. Duncan T, Sebar B, Lee J, Duff C. Mapping the spatial and anective composition of care in a drug consumption room in Germany. Social \& Cultural Geography. 2019;1-20. doi: 10.1080/14649365.2019.1610487.

52. Bardwell G, Strike C, Altenberg J, Barnaby L, Thomas Kerr T. Implementation contexts and the impact of policing on access to supervised consumption services in Toronto, Canada: a qualitative comparative analysis. Harm Reduction Journal. 2019;16(1):30.

53. Zuffa G. Cocaína. Hacia un modelo basado en la autorregulación: Nuevas tendencias en la reducción de daños. Serie reforma legislativa en materia de drogas. Amsterdam: Transnational Institute; 2014. 
54. Small W, Ainsworth L, Wood E, Kerr T. IDU perspectives on the design and operation of North America's first medically supervised injection facility. Substance Use \& Misuse. 2011;46(5):561-568. doi: 10.3109/10826084.2010.517714.

55. Llort A, Ferrando S, Borrás T, Purroy I. El doble estigma de la mujer consumidora de drogas: estudio cualitativo sobre un grupo de auto apoyo de mujeres con problemas de abuso de sustancias. Alternativas: Cuadernos de Trabajo Social. 2013;20:9-22

56. \#CocaReguladaPazGarantizada [Internet] 2017 [citado 20 jul 2020]. Disponible en: https://cocaregulada.com/

57. Duncan T, Sebar B, Lee J, Duff C. Atmospheres of engagement within a German drug consumption room. Social Science \& Medicine. 2020;253:112922. doi: 10.1016/j.socscimed.2020.112922.

58. Rance J, Fraser S. Accidental intimacy: Transformative emotion and the Sydney Medically Supervised Injecting Centre. Contemporary Drug Problems. 2011;38(1):121 145. doi: 10.1177/009145091103800106.

59. Romo N, Poo M, Ballesta R, PEPSA team. From illegal poison to legal medicine: a qualitative research in a heroin-prescription trial in Spain. Drug and Alcohol Review. 2009;28(2):186-195. doi: 10.1111/j.14653362.2008.00015.x.

60. Oviedo-Joekes E, Marchand K, Lock K, Chettiar J, Marsh DC, Brissette S, Anis AH, Schechter MT. A chance to stop and breathe: participants' experiences in the North American Opiate Medication Initiative clinical trial. Addiction Science \& Clinical Practice. 2014;9(1):21. doi: 10.1186/1940-0640-9-21.

61. Pauly B, Reist D, Belle-Isle L, Schactman C. Housing and harm reduction: what is the role of harm reduction in addressing homelessness. International Journal on Drug Policy. 2013;24(4):284-290. doi: 10.1016/j. drugpo.2013.03.008.

62. Pauly BM, Gray E, Perkin K, Chow C, Vallance K, Krysowaty B, Stockwell T. Finding safety: a pilot study of managed alcohol program participants' perceptions of housing and quality of life. Harm Reduction Journal. 2016;13:15

63. Llort A. El pájaro está en el nido: Cocaína, cultura y salud, una etnografía del consumo de cocaína en Reus. Tarragona: Publicacions de la Universitat Rovira i Virgili; 2019.

64. Clua R. Estilos de vida de los usuarios de las salas de consumo higiénico de Barcelona. Health and Addictions/Salud y Drogas. 2018;18(2):79-89.

65. Duff C. The pleasure in context. International Journal on Drug Policy. 2008;19(5):384-392. doi: 10.1016/j. drugpo.2007.07.003.

66. Gonçalves JR, Nappo SA. Factors that lead to the use of crack cocaine in combination with marijuana in Brazil: a qualitative study. BMC Public Health. 2015;15:706.

67. Rhodes T, Stimson GV, Moore D, Bourgois P. Qualitative social research in addictions publishing: Creating an enabling journal environment. International Journal on Drug Policy. 2010;21(6):441-444. doi: 10.1016/j. drugpo.2010.10.002.

68. Martínez Oró DP. Sin pasarse de la raya: La normalización de los consumos de drogas. Barcelona: Bellaterra; 2015.

69. Bourgois P. Anthropology and epidemiology on drugs: Cross-Methodological and theoretical dialogue. International Journal on Drug Policy. 2002;13(4):259269. doi: 10.1016/S0955-3959(02)00115-9.

70. Lopez A, Bourgois P, Wenger L, Lorvick J, Martinez A, Kral AH. Interdisciplinary mixed methods research with structurally vulnerable populations: Case studies of injection drug users in San Francisco. International Journal on Drug Policy. 2013;24(2):101-109. doi 10.1016/j.drugpo.2012.12.004.

71. Romaní O. La epidemiología sociocultural en el campo de las drogas: contextos, sujetos y sustancias. En: Haro JA, (coord.). Epidemiología sociocultural: Un diálogo en torno a su sentido, métodos y alcances. Buenos Aires: Lugar Editorial; 2010. p. 89-113. 